\title{
PADRÕES ESPACIAIS NA REGENERAÇÃO DE Ocotea odorifera NA FLORESTA NACIONAL DE IRATI, PR
}

\author{
Cilmar Antônio Dalmaso ${ }^{1}$, Mario Takao Inoue ${ }^{2}$, Paulo Costa de Oliveira Filho ${ }^{3}$, \\ Vânia Rossetto Marcelino ${ }^{4}$ \\ ${ }^{1}$ Eng. Florestal, M.Sc., UNICENTRO, Irati, PR, Brasil - cilmard@ gmail.com \\ ${ }^{2}$ Eng. Florestal, Dr., Depto. de Engenharia Florestal, UNICENTRO, Irati, PR, Brasil - martakino@gmail.com \\ ${ }^{3}$ Eng. Florestal, Dr., Depto. de Engenharia Ambiental, UNICENTRO, Irati, PR, Brasil - paulocostafh@ gmail.com \\ ${ }^{4}$ Ecóloga, Dra ${ }^{\text {., }}$ Depto. de Engenharia Florestal, UNICENTRO, Irati, PR, Brasil - vania@irati.unicentro.br \\ Recebido para publicação: 12/09/2012 - Aceito para publicação: 05/05/2013
}

\begin{abstract}
Resumo
Este trabalho teve o objetivo de analisar e descrever os padrões espaciais e as características qualiquantitativas da regeneração da espécie arbórea Ocotea odorifera (Vell.) Rohwer (canela-sassafrás) num fragmento de Floresta Ombrófila Mista localizado na Floresta Nacional de Irati, região centrosul do estado do Paraná. Para tanto, foram selecionados três hectares de amostra para coleta de informações sobre altura, diâmetro e localização de todos os indivíduos de canela-sassafrás com altura total $\geq 30 \mathrm{~cm}$. Análises univariadas por meio da função $K$ de Ripley foram realizadas, para descrever os padrões espaciais dos indivíduos em fases distintas de regeneração e das árvores adultas dessa espécie com diferentes tamanhos. A espécie apresentou em geral um padrão espacial agregado para a regeneração natural em todas as escalas analisadas, sugerindo ser um padrão característico da espécie nas fases iniciais. A mudança do padrão agregado para aleatório foi comprovada com a análise espacial para as diferentes classes de tamanho dos indivíduos de canela-sassafrás. Algumas inferências foram feitas em relação aos processos subjacentes, que podem estar associados com os resultados. É possível afirmar que a espécie apresenta potencialidades para o manejo e a conservação com excelente capacidade de regeneração natural.
\end{abstract}

Palavras-chave: Floresta com Araucária; autoecologia; estatística espacial; canela-sassafrás.

\begin{abstract}
Spatial pattenrs for Ocotea odorifera regeneration in National Forest, Irati, Pr. The present research aimed to analyze and describe spatial patterns and quali-quantitative features of natural regeneration of tree species of Ocotea odorifera (Vell.) Rohwer (canela-sassafrás) in a fragment of Mixed Ombrophyllous Forest (Araucarian Forest) located at the National Forest of Irati, Paraná State, Southern Brazil. Three plots of one hectar each were sampled and height, diameter and location informations were collected for all plants of canela-sassafrás with total height $\geq 30 \mathrm{~cm}$. Univariate analyzes using $K$-function of Ripley were done in order to describe spatial patterns of plants at different regeneration phases as well as of canela-sassafrás adults. Generally, this species presented an aggregated spatial pattern at all considered scales of regeneration, suggesting to be a typical pattern at initial phases of this species. The change from aggregated to random spatial pattern was tested analyzing different size classes of canela-sassafrás. Some inferences were made considering subjacent processes that could be associated to obtained results. It is possible to say that the studied species presents potencialities for its management and conservation with excellent natural regeneration capacity.
\end{abstract}

Keywords: Araucaria Forest; auto-ecology; spatial statistics; canela-sassafrás.

\section{INTRODUÇÃO}

A canela-sassafrás (Ocotea odorifera (Vell.) Rohwer) pertence à família Lauraceae e é endêmica do Brasil. No passado, sua madeira foi muito visada pelas qualidades em diversos usos e principalmente devido à alta concentração do óleo essencial, cujo principal componente químico é o safrol (RIZZINI, 1981; REITZ et al., 1983; CARVALHO, 1994; INOUE et al., 1984), utilizado na fabricação de inseticidas biodegradáveis, cosméticos e produtos farmacêuticos (AZAMBUJA, 2011). A química desse 
composto natural tem sido largamente estudada, sendo tema de diversas dissertações e teses realizadas em diferentes programas de pós-graduação do País (BARREIRO; FRAGA, 1999). A madeira da canelasassafrás possui excelentes propriedades técnicas e por isso pode ser utilizada na marcenaria de luxo, dormentes, construção civil e também na construção naval (VATTIMO, 1979). Atualmente, a exploração da espécie está restrita por lei, sendo seu uso permitido somente em casos excepcionais, como obras de utilidade pública ou para árvores isoladas que acarretem risco ao patrimônio público ou particular e à segurança da população. A não sustentabilidade do modelo de produção utilizado (extrativismo) foi determinante para que a espécie fosse levada a uma condição crítica de sobrevivência, com implicações significativas para sua exploração comercial (OLTRAMARI, 2002). Considerando a situação atual de risco de extinção, são poucos os estudos realizados sobre a regeneração e a silvicultura da espécie (INOUE; PUTTON, 2007; INOUE et al., 2004).

Levando-se em consideração a importância da espécie, tanto sob o ponto de vista comercial como igualmente o seu valor ecológico, objetivou-se neste estudo caracterizar a regeneração natural da canelasassafrás por meio de análise de padrões espaciais dos indivíduos de diferentes classes de tamanho.

\section{REVISÃO DA LITERATURA}

Compreender como estão organizadas as comunidades e populações vegetais desde o ponto de vista da sua estrutura espacial é fundamental para definir estratégias de manejo e conservação dos recursos florestais. Padrões espaciais de plantas em comunidades naturais carregam informações sobre os processos que operaram no passado e que formam o modelo no qual os processos terão lugar no futuro (LAW et al., 2009). O padrão espacial apresentado pelos indivíduos de uma espécie vegetal é gerado por interações entre fatores bióticos e abióticos complexos, sendo uma questão importante em estudos ecológicos. De acordo com Silva et al. (2009), há um crescente interesse em estudar padrões espaciais em comunidades vegetais com base no pressuposto de que, para entendê-las, deve-se descrever e quantificar as características espaciais e temporais e relacioná-las a processos subjacentes, como crescimento, competição, reprodução e mortalidade. Para Capretz (2004), a escolha de áreas para conservação de espécies de interesse deve levar em consideração a caracterização de seu padrão espacial para a definição do tamanho adequado da população a ser conservada.

O padrão espacial de uma espécie refere-se à distribuição dos seus indivíduos na superfície da floresta. O termo "padrão" nesse caso é utilizado para expressar a organização e o ordenamento espacial dos indivíduos (MATTEUCCI; COLMA, 1982). O termo "distribuição" tem significado preciso em estatística, pois denota a forma como se dividem as classes de tamanho dos possíveis valores de uma variável (CRUZ; RÍOS, 2003).

Conforme Begon et al. (2006), podem-se reconhecer três padrões principais de dispersão, embora eles formem parte de um continuum, sendo eles: aleatório (ocorre quando existe uma probabilidade igual de um organismo ocupar qualquer ponto no espaço, independentemente da posição de qualquer outro); regular (também chamada de distribuição uniforme, ocorre quando um indivíduo tem uma tendência para evitar outros indivíduos, e o resultado é que os indivíduos estão mais uniformemente espaçados do que o esperado por acaso); e agregada (também chamada de distribuição contagiosa ou agrupada, que ocorre quando os indivíduos tendem a ser atraídos entre si, ou quando são mais propensos a sobreviver em partes específicas do ambiente, ou quando a presença de um indivíduo atrai ou dá origem a um outro perto dele; o resultado é que os indivíduos estão mais próximos do que o esperado por acaso). Grande parte dos autores concorda que a distribuição espacial aleatória é rara em populações de plantas, assim como a distribuição uniforme, e que a maioria das populações tem algum grau de agregação dos indivíduos no espaço (HORVITZ; LE CORFF, 1993; LEGENDRE; MELLO, 2002).

Os métodos frequentemente utilizados na ecologia florestal para as análises dos padrões espaciais são baseados em distâncias ou áreas e tratam apenas da simples contagem de indivíduos dentro de parcelas ou da procura pelo vizinho mais próximo da mesma espécie. Para Sterner et al. (1986), o uso desses métodos provoca a perda de muita informação, uma vez que tais métodos só conseguem detectar o padrão espacial naquela escala particular em que os dados foram coletados, como tamanho da parcela ou distância entre os pontos. As informações em escalas maiores são ignoradas. Como alternativa para detectar e descrever o padrão espacial em diversas escalas, a estatística espacial apresenta algumas possibilidades. A estatística espacial trata de analisar dados espaciais com o objetivo de mensurar propriedades e 
relacionamentos considerando a localização espacial do fenômeno em estudo, ou seja, permite estudar, explorar e modelar fenômenos geográficos (DRUCK et al., 2004; GOODCHILD; HAINING, 2004). Um caso em particular para avaliar o padrão de pontos em diversas escalas é a função $K$ de Ripley (RIPLEY, 1977, 1981; BATISTA; MAGUIRE, 1998). A noção gráfica intuitiva do que está consagrado na função $K$ pode ser imaginada como um conjunto de círculos concêntricos, centrados em um evento com um espaçamento definido onde o número acumulado de eventos em cada uma dessas distâncias "bandas" é contado (BAILEY; GATRELL, 1995). Na prática, é necessário introduzir um fator para corrigir o "efeito de borda”, porque os pontos fora dos limites da área de estudo não são levados em conta.

Ocotea odorifera (canela-sassafrás) é uma espécie arbórea de interesse para a conservação e produção. Pode ser encontrada nos biomas Amazônia, Cerrado e Mata Atlântica, principalmente nos estados do Norte (PA), Nordeste (PE, BA), Sudeste (MG, ES, SP, RJ) e Sul (PR, SC, RS) (QUINET et al., 2012), entre as coordenadas $8^{\circ} 45^{\prime} \mathrm{S}$ (Pernambuco) a $31^{\circ} 12^{\prime} \mathrm{S}$ (Rio Grande do Sul), e de $33^{\circ} 52^{\prime}$ a $59^{\circ} 09^{\prime}$ de longitude oeste (SALOMÃO et al., 1992), com variação altitudinal de $15 \mathrm{~m}$ em Santa Catarina a $1.300 \mathrm{~m}$ de altitude em Minas Gerais (CARVALHO, 2005). No estado do Paraná, apresentava ampla dispersão, ocorrendo na Floresta Atlântica, nas Florestas com Araucária e nas formações tropicais do norte do estado (KEIL et al., 2009).

A relevância deste estudo se deve principalmente aos compostos e propriedades extraídas dessa espécie, que possuem alto valor agregado e com propriedades farmacológicas importantes. Atualmente, o Brasil importa safrol utilizado na fabricação de inseticidas biodegradáveis, cosméticos e produtos farmacêuticos. Espera-se, com este estudo, contribuir para o conhecimento da autoecologia e gerar informações úteis para o manejo e conservação da espécie.

\section{MATERIAL E MÉTODOS}

\section{Área de estudo}

A área de estudo (Figura 1) está localizada na Floresta Nacional (FLONA) de Irati, região centro-sul do estado do Paraná, entre os municípios de Fernandes Pinheiro, Imbituva, Irati e Teixeira

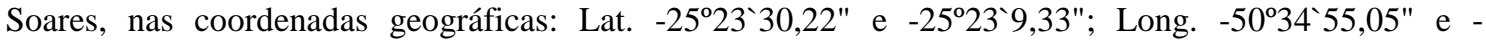
$50^{\circ} 34^{2} 26,49 "$. A FLONA de Irati é uma Unidade de Conservação de Uso Sustentável (administrada pelo Instituto Chico Mendes de Conservação da Biodiversidade - ICMBio) e compreende 3.618,21 hectares, com 43,82\% de sua área sendo ocupados por cobertura florestal nativa (MAZZA, 2006).

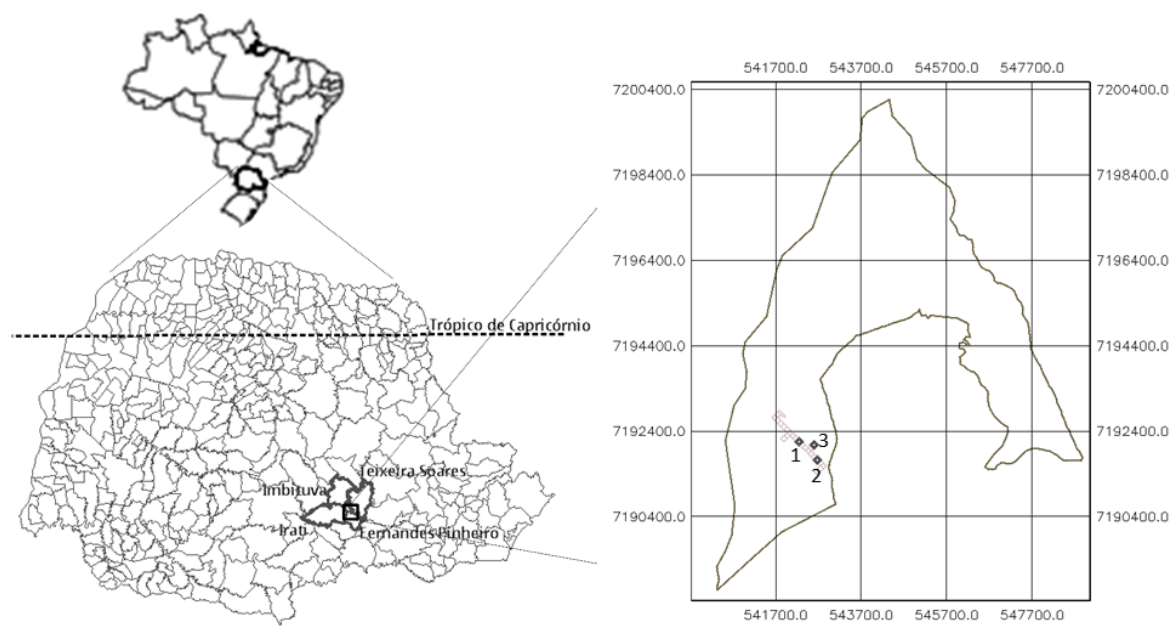

Figura 1. Localização geográfica da FLONA de Irati, entre os municípios de Fernandes Pinheiro, Imbituva, Irati e Teixeira Soares, no estado do Paraná, Brasil. Destaque para os três Blocos (1, 2 e 3) do trabalho.

Figure 1. Geographic location of Irati's FLONA (National Forest), within the counties of Fernandes Pinheiro, Imbituva, Irati and Teixeira Soares, in Paraná State, Brazil. Highlighted, the three studied sample blocks (1,2 and 3). 
A altitude na FLONA de Irati varia entre 788 e 899 m, sendo que a área de estudo está em um local elevado, com boa drenagem. O clima da região, de acordo com o sistema de Köppen, é do tipo Cfb, ou seja, subtropical úmido sem estação seca, com estações de verão e inverno bem definidas.

Os solos que predominam são profundos, porosos e argilosos, do grupo Latossolo Vermelho distrófico, o que lhes confere porosidade e livre drenagem da água (EMBRAPA, 1999).

A área de estudo faz parte de um experimento multidisciplinar em 25 hectares contínuos de Floresta Ombrófila Mista (FOM) (FIGUEIREDO FILHO et al., 2010). Segundo a classificação de Veloso et al. (1991), no sítio de amostragem ocorre a fitofisionomia de Floresta Ombrófila Mista Montana. Para esta pesquisa, foram escolhidos três blocos com dimensões de 100 x $100 \mathrm{~m}$ e alta densidade de árvores adultas de canela-sassafrás, onde supostamente deve existir uma maior quantidade de regenerantes para análise dos padrões e interações espaciais. Cada um desses blocos foi dividido em quatro parcelas com dimensões de 50 x $50 \mathrm{~m}$.

\section{Coleta de dados}

Em cada bloco, todos os indivíduos de canela-sassafrás com mais de $30 \mathrm{~cm}$ de altura total foram mapeados por coordenadas cartesianas locais e mensurados quanto à altura total. Ainda, foram coletados os diâmetros (DAP) dos indivíduos acima de 3 metros. As alturas até 3 metros foram mensuradas com trena de 5 metros, e as alturas maiores foram estimadas. Para facilitar a numeração e o mapeamento dos representantes da espécie, foram instaladas cinco faixas de 0,05 ha $(10 \times 50 \mathrm{~m}) \mathrm{em}$ cada parcela, delimitadas com fita zebrada.

\section{Caracterização da estrutura horizontal e vertical da canela-sassafrás}

Inicialmente, os indivíduos de cada amostra foram classificados nas duas categorias listadas:

1. Adultas - plantas maiores, a partir de $10 \mathrm{~cm}$ de DAP;

2. Regenerantes - plantas menores que $10 \mathrm{~cm}$ de DAP.

A estrutura diamétrica das árvores adultas de canela-sassafrás foi descrita pelas distribuições de frequência do número de árvores para classes de diâmetro de $10 \mathrm{~cm}$.

A estrutura vertical para a regeneração foi descrita usando-se intervalos de altura adaptados de Dubois (1980) e Cruz e Ríos (2003). Com base nessa classificação, as categorias consideradas neste trabalho foram:

1.1. Mudas inferiores (U1) - com alturas de 30 a $150 \mathrm{~cm}$;

1.2. Mudas superiores (U2) - com alturas $>150 \mathrm{~cm} \mathrm{e} \leq 300 \mathrm{~cm}$;

1.3. Estabelecidas (E) - com alturas superiores a $300 \mathrm{~cm}$ e inferiores a $5 \mathrm{~cm}$ de DAP;

1.4. Arvoretas (L) - corresponde aos indivíduos com diâmetros entre 5 e $10 \mathrm{~cm}$.

\section{Análise dos padrões espaciais}

Foram utilizados métodos com base na análise das distâncias entre pontos da função $K$ (RIPLEY, 1977 ; 1981; 1988). Os dados espaciais consistiram em mapas de pontos elaborados a partir das coordenadas cartesianas de cada bloco. As tabelas foram digitadas e editadas em planilhas do Microsoft Office Excel 2007 e salvas em arquivo de texto formato txt. Os arquivos de texto foram importados para o programa computacional "R" versão 2.15.0 (R, 2012). O pacote utilizado no R foi o "spatstat" versão 1.27-0 (BADDELEY; TURNER, 2005).

Os padrões espaciais foram descritos por meio da função $K$, incorporada a correção de borda de Ripley (1977) e a transformação de raiz quadrada, $L$. A escala de distância utilizada neste estudo vai de 0 a $25 \mathrm{~m}$, de modo a proporcionar estimativas não viciadas. Em todos os casos, o modelo nulo (hipótese de aleatoriedade espacial) foi gerado com 500 simulações Monte Carlo para $\alpha=0,01$ com os limites de confiança.

Foram realizadas análises univariadas para 12 categorias de tamanho de canela-sassafrás, visando avaliar a evolução do padrão espacial, sendo 9 classes de regeneração e 3 classes de tamanho de árvores adultas, conforme segue: 1 - indivíduos (ind.) de 30 a 49 cm; 2 - ind. de 50 a 69 cm; 3 - ind. de 70 a $89 \mathrm{~cm} ; 4$ - ind. de 90 a $109 \mathrm{~cm} ; 5$ - ind. de 110 a $129 \mathrm{~cm} ; 6$ - ind. de 130 a $149 \mathrm{~cm} ; 7$ - ind. de 150 a $299 \mathrm{~cm} ; 8$ - ind. de $300 \mathrm{~cm}$ de altura total e DAP $\leq 5 \mathrm{~cm} ; 9$ - ind. entre 5 e $10 \mathrm{~cm}$ de DAP; 10 - DAP de 10 a 14,9 cm; 11 - DAP de 15 a 19,9 cm; 12 - DAP $\geq 20 \mathrm{~cm}$. Como o número de indivíduos nas classes superiores (DAP>20) é insuficiente para realizar a função $K$, elas foram agrupadas em apenas uma classe. 


\section{RESULTADOS E DISCUSSÃO}

No total, foram mensurados 5.549 indivíduos de canela-sassafrás com altura maior que $30 \mathrm{~cm}$. O Bloco 1, com a maior densidade, apresentou um total de 2.929 indivíduos, o Bloco 2 apresentou um total de 871 indivíduos e o Bloco 3 contabilizou 1.749 indivíduos. A distribuição das frequências nas categorias em regeneração (DAP $<10 \mathrm{~cm}$ ) e adultas (DAP $\geq 10 \mathrm{~cm}$ ) pode ser observada na figura 2 . Observa-se a forma do "J-invertido" dos histogramas, como é esperado em florestas heterogêneas. Esse padrão denota uma comunidade com potencial de regeneração e com muitos indivíduos jovens em relação ao número de indivíduos adultos (RAMOS et al. 2008), prováveis plantas matrizes ou porta-sementes.

$\mathrm{O}$ grande número de adultos pertencentes às primeiras classes da distribuição (Figura 2B; Figura 2D e Figura $2 \mathrm{~F}$ ) e o decréscimo relativamente gradual com a amplitude em diâmetro conferem à população de Ocotea odorifera um bom estoque de indivíduos. Péllico Netto et al. (2007) também encontraram uma configuração diamétrica na forma de "J-invertido" (decrescente) para a canela-sassafrás em diversas áreas estudadas na Região Metropolitana de Curitiba, PR.
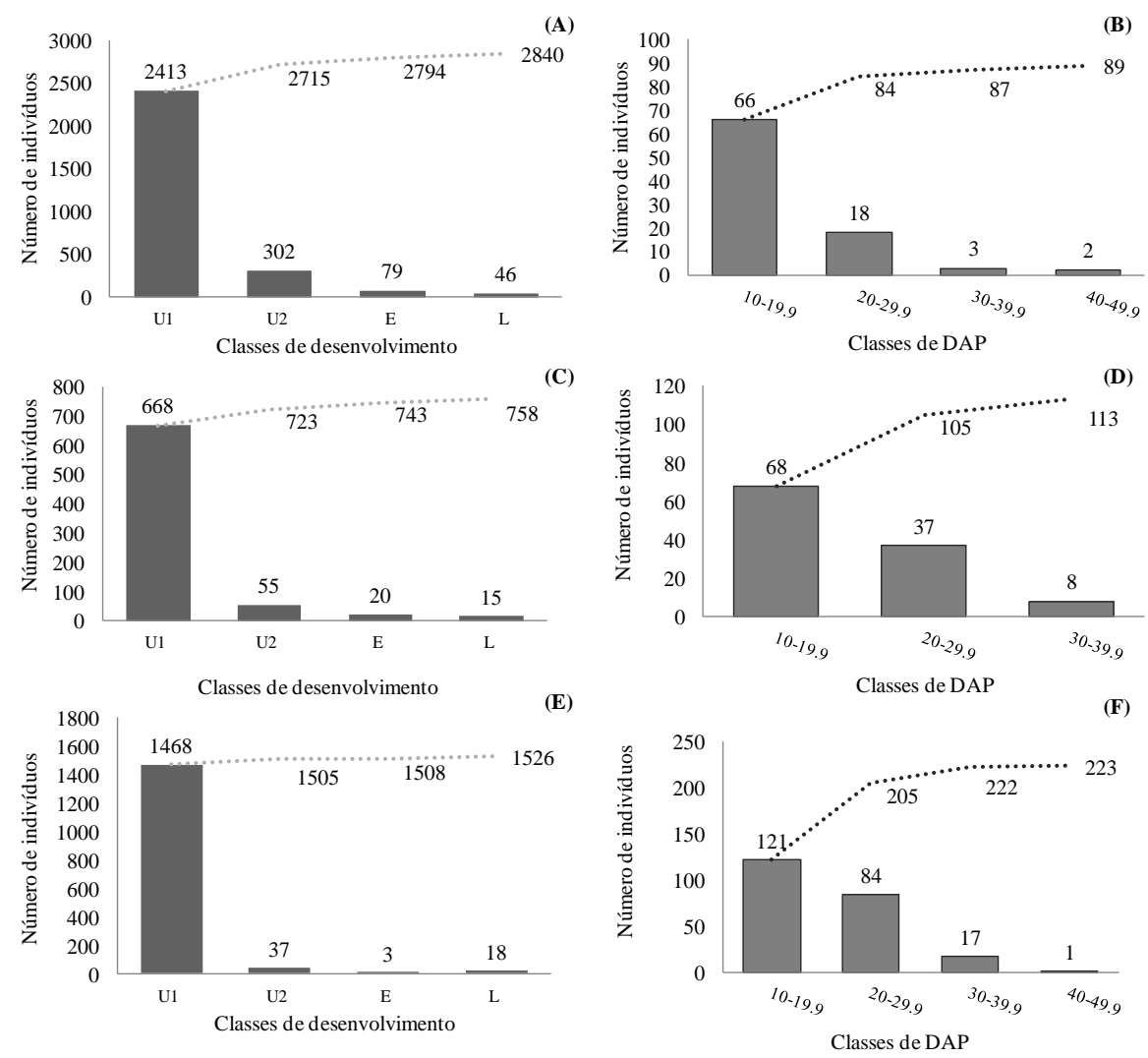

Figura 2. Frequência absoluta e acumulada da distribuição vertical para a regeneração de canelasassafrás em classes de desenvolvimento (esquerda) e para distribuição diamétrica $(\mathrm{cm})$ dos indivíduos adultos da canela-sassafrás (direita). FLONA de Irati, PR. Bloco 1 (A) e (B); Bloco 2 (C) e (D); Bloco 3 (E) e (F).

Figure 2. Absolute and accumulated frequencies of vertical distribution of canela-sassafrás regeneration, in growth classes (left) and as diametric distribution of adults trees (right, in centimeters). FLONA of Irati, PR. Block 1 (A) and (B); Block 2 (C) and (D); Block 3 (E) and (F).

A partir de dados de 2011, fornecidos pelo Laboratório de Manejo Florestal da UNICENTRO, foram obtidos alguns índices referentes à estrutura horizontal nos blocos avaliados. A canela-sassafrás teve uma densidade relativa alta na comunidade $(15,98 \%, 18,43 \%$ e $34,31 \%$, respectivamente, para Bloco 1, Bloco 2 e Bloco 3), considerando que um grande número de espécies com poucos indivíduos 
ocorreu em todos os blocos. A canela-sassafrás se destacou também com 9,42\%, 12,12\% e 24,77\% da área basal (dominância relativa) no Bloco $1\left(2,58 \mathrm{~m}^{2}\right)$, Bloco $2\left(3,60 \mathrm{~m}^{2}\right)$ e Bloco $3\left(7,78 \mathrm{~m}^{2}\right)$, respectivamente. Esses aspectos destacam características desejáveis em programas de manejo e conservação in situ de recursos genéticos em florestas naturais (RAMOS et al., 2008).

Um número bastante expressivo de canelas-sassafrás foi encontrado na área de estudo, estando relacionado à capacidade de regeneração da espécie nesse sítio. Carvalho (2003) mencionou que $O$. odorifera é exigente em solos, sendo considerada espécie indicadora de alta fertilidade química, tendo crescido melhor em solos férteis, profundos, com drenagem boa e textura argilosa.

\section{Padrões espaciais para classes de tamanho}

Os resultados são descritos por meio dos gráficos em que o eixo das ordenadas corresponde ao valor estimado e transformado de $K$ ( $L$ estimado) e o eixo das abscissas corresponde às distâncias em metros (escala). Num padrão aleatório, a função empírica (L estimado) deve estar dentro dos limites de confiança. Quando a função empírica ("L estimado" - linha sólida) supera o valor teórico esperado (envelope com área na cor cinza) do limite de confiança, assumindo que os pontos são completamente aleatórios, significa agrupamento; já no padrão regular a função (L estimado) está abaixo do envelope do limite de confiança. Com os resultados, é possível observar como evolui o padrão espacial ao longo do ciclo de vida das árvores, conforme figuras 3,4 e 5.
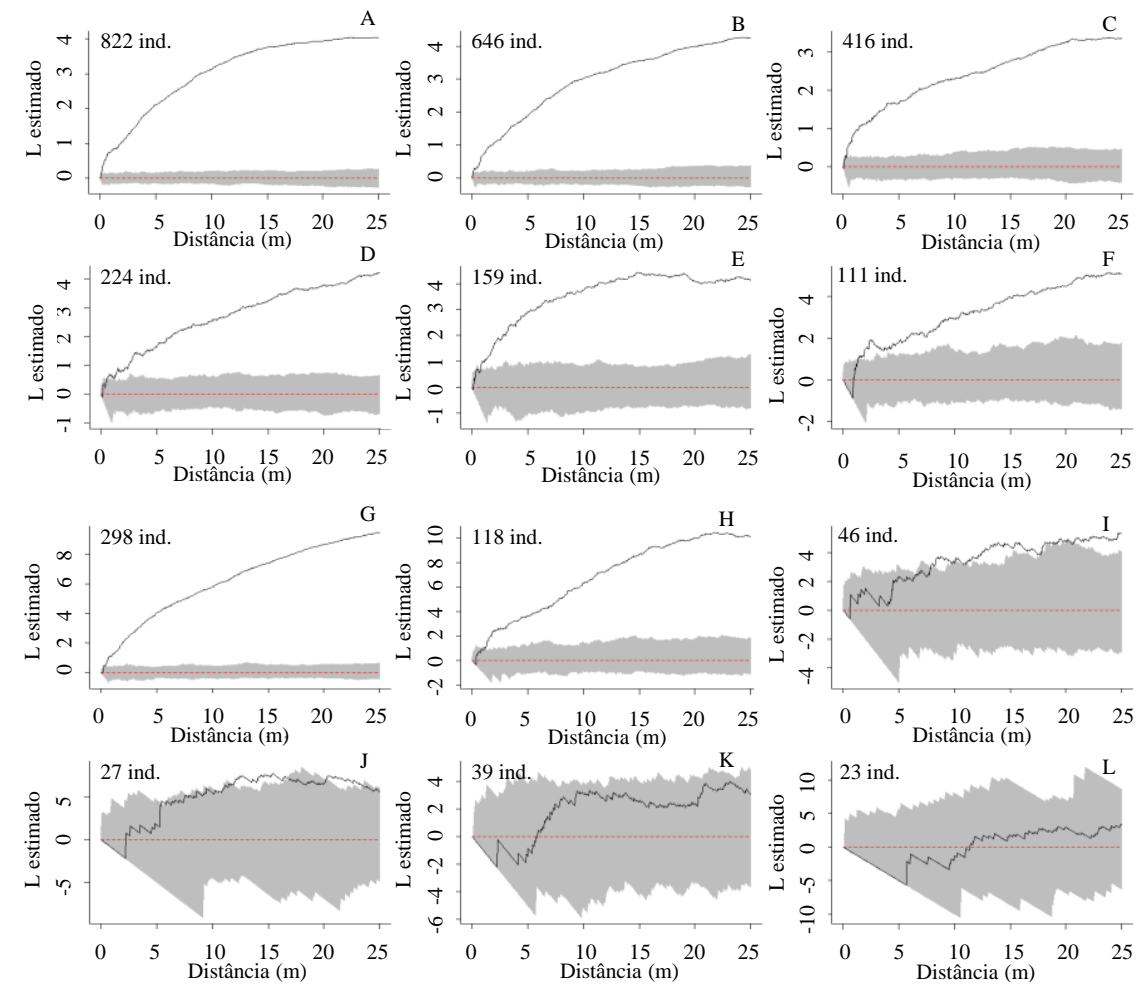

Figura 3. Função $K$-univariada ( $L$ estimado) para diferentes categorias de altura total (cm) dos indivíduos regenerantes de canela-sassafrás no Bloco 1 na FLONA de Irati, PR. Classes dos indivíduos regenerantes (altura total em cm): (A) 30-49; (B) 50-69; (C) 70-89; (D) 90-109; (E) 110-129; (F) 130-149; (G) 150-299; (H) $\geq 300$ e DAP(cm) $\leq 5$; (I) DAP(cm) 5-10. Classes dos indivíduos adultos (DAP em cm): (J) 10-15; (K) 15-20; (L) $\geq 20$.

Figure 3. Values of univariated $K$-function (estimated $L$ ) for different total height groups $(\mathrm{cm})$ of canelasassafrás regeneration at Block 1 in the FLONA of Irati, PR. Classes of regenerative individuals (total height cm): (A) 30-49; (B) 50-69; (C) 70-89; (D) 90-109; (E) 110-129; (F) 130-149; (G) 150-299; (H) $\geq 300$ e DBH (cm) $\leq 5$; (I) DBH (cm) 5-10. Classes for adults (DBH cm): (J) 10-15; (K) 15-20; (L) $\geq 20$. 
Foi observada uma tendência geral da canela-sassafrás em apresentar distribuição fortemente agrupada em todas as escalas e classes no início do desenvolvimento (Figuras 3, 4 e 5 "A; B; C; D; E; F; G; H e I"). Para os indivíduos adultos, as três classes de diâmetro apresentaram padrão espacial aleatório para os três blocos (Figuras 3, 4 e 5 "J; K e L").

Para Silva et al. (2009), a formação de padrões agregados para os indivíduos mais jovens está relacionada à dispersão de sementes em curta distância e à ocorrência de partes mais favoráveis de um hábitat, ainda que, devido à eliminação por competição intraespecífica, o padrão possa tender a ser aleatório ou uniforme. Segundo Condit et al. (2000), o padrão agregado ocorre principalmente em razão do maior número de sementes e plântulas próximas da planta-mãe e do grau de heterogeneidade ambiental. Apesar de a canela-sassafrás ser tolerante à sombra nas fases iniciais de desenvolvimento, os diferentes níveis de intensidade luminosa proporcionados pelo mosaico do dossel e clareiras podem aumentar a sobrevivência de plântulas em locais específicos. Além da luminosidade, relevo, nutrientes, umidade e solo, entre outros aspectos, podem gerar a heterogeneidade ambiental na FOM.
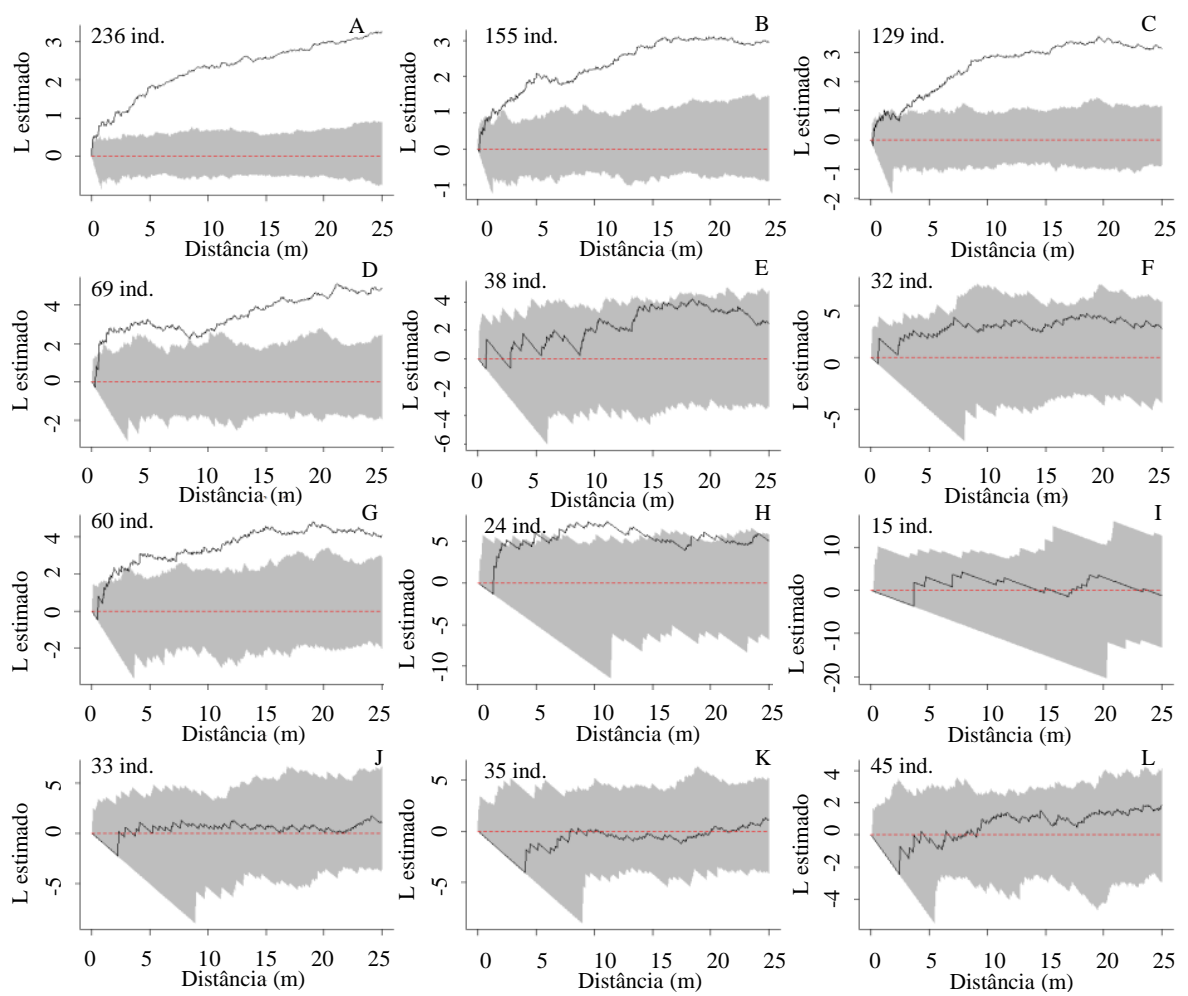

Figura 4. Função $K$-univariada ( $L$ estimado) para diferentes categorias de altura total $(\mathrm{cm})$ dos indivíduos regenerantes de canela-sassafrás no Bloco 2 na FLONA de Irati, PR. Classes dos indivíduos regenerantes (altura total em cm): (A) 30-49; (B) 50-69; (C) 70-89; (D) 90-109; (E) 110-129; (F) 130-149; (G) 150-299; (H) $\geq 300$ e DAP(cm) $\leq 5$; (I) DAP (cm) 5-10. Classes dos indivíduos adultos (DAP cm): (J) 10-15; (K) 15-20; (L) $\geq 20$.

Figure 4. Values of univariated $K$-function (estimated $L$ ) for different total height groups $(\mathrm{cm})$ of canelasassafrás regeneration at Block 2 in the FLONA of Irati, PR. Classes of regenerative individuals (total height cm): (A) 30-49; (B) 50-69; (C) 70-89; (D) 90-109; (E) 110-129; (F) 130-149; (G) 150-299; (H) $\geq 300$ e DBH (cm) $\leq 5$; (I) DBH (cm) 5-10. Classes for adults (DBH cm): (J) 10-15; (K) 15-20; (L) $\geq 20$.

Conforme as plântulas vão passando pelos estádios de desenvolvimento, vão sofrendo diferentes taxas de mortalidade, e o padrão espacial dos indivíduos adultos, originado com a dispersão de sementes, acaba sendo moldado de diferentes formas pelas taxas de mortalidade em cada estádio (MELLO, 2002). 
Para Crawley (1986), o padrão espacial de indivíduos maduros reflete o padrão espacial de recrutamento e a influência de fatores de mortalidade, que podem diferir em intensidade entre locais distintos. Para Silva et al. (2009), o padrão espacial de adultos tende a ser menos agregado do que o de plântulas para espécies com alta taxa de mortalidade dependente de densidade.
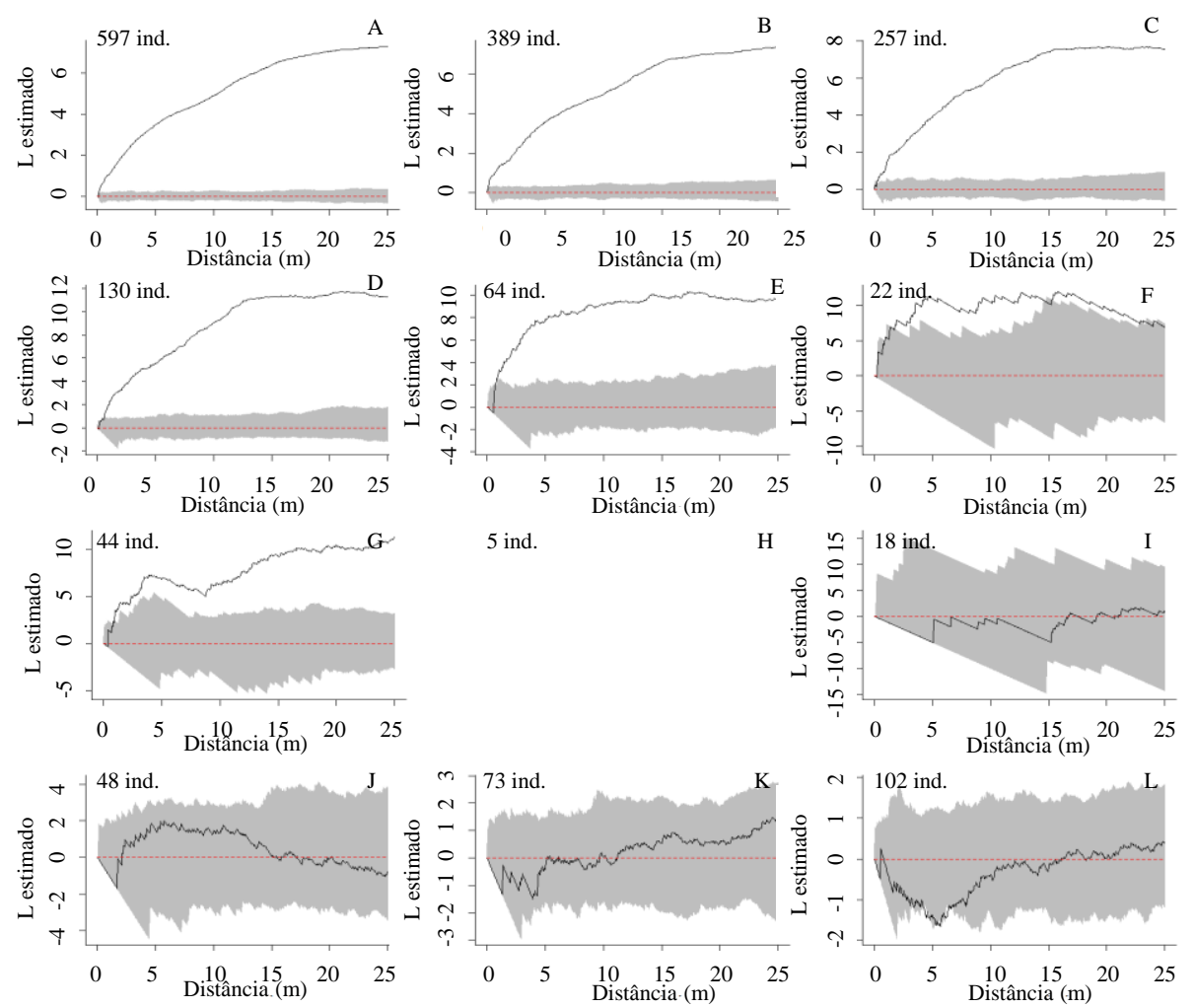

Figura 5. Função $K$-univariada ( $L$ estimado) para diferentes categorias de altura total (cm) dos indivíduos regenerantes de canela-sassafrás no Bloco 3 na FLONA de Irati, PR. Classes dos indivíduos regenerantes (altura total cm): (A) 30-49; (B) 50-69; (C) 70-89; (D) 90-109; (E) 110-129; (F) 130-149; (G) 150-299; (H) $\geq 300$ e DAP $(\mathrm{cm}) \leq 5$; (I) DAP (cm) 5-10. Classes dos indivíduos adultos (DAP em cm): (J) 10-15; (K) 15-20; (L) $\geq 20$.

Figure 5. Values of univariated $K$-function (estimated $L$ ) for different total height groups $(\mathrm{cm})$ of canelasassafrás regeneration at Block 3 in the FLONA of Irati, PR. Classes of regenerative individuals (total height cm): (A) 30-49; (B) 50-69; (C) 70-89; (D) 90-109; (E) 110-129; (F) 130-149; (G) 150-299; (H) $\geq 300$ e DBH (cm) $\leq 5$; (I) DBH (cm) 5-10. Classes for adults (DBH cm): (J) 10-15; (K) 15-20; (L) $\geq 20$.

Observou-se que é necessário um número mínimo de indivíduos para rodar a Função K. O gráfico $\mathrm{H}$ da figura 5 não foi possível analisar, pois 5 observações inviabilizam a análise espacial dos dados do bloco, lembrando que o valor que inviabiliza a análise é proporcional à área da amostra. Neste estudo, utilizando as amostras de $100 \times 100 \mathrm{~m}$, os gráficos com menos de 20 observações se apresentam mais duvidosos.

Quando há densas agregações locais e a demanda por recursos aumenta consideravelmente, ocorre competição intraespecífica (MELLO, 2002). Essa competição induz uma mortalidade dependente de densidade, que tende a diminuir diferenças locais de densidade (CRAWLEY, 1986). Nesse caso, o padrão aleatório pode ser mais frequente com o aumento da idade. O padrão agregado dos indivíduos regenerantes e aleatório das árvores adultas sustenta, portanto, a hipótese de que a canela-sassafrás apresenta altas taxas de mortalidade dependente de densidade.

Lehn et al. (2008), analisando as populações de jovens e adultos de Trichilia elegans A. Juss., 
espécie típica de sub-bosque, também constataram que os indivíduos jovens se encontram mais agrupados e que o grau de agregação tende a diminuir durante o desenvolvimento ontogenético.

Os padrões aleatórios que predominaram para as árvores adultas podem implicar homogeneidade ambiental e/ou padrões comportamentais não seletivos (MATTEUCCI; COLMA, 1982). Para Crawley (1986), quando a mortalidade é dependente de fatores abióticos, a tendência é que a população esteja mais concentrada em manchas de hábitat favorável, e a maioria das mortes ocorrerá nas bordas da distribuição espacial. Conforme esse autor, a distribuição espacial de plântulas de uma determinada espécie depende da interação entre quatro fatores. Primeiro, a forma como as plantas-mãe produtoras de sementes estão localizadas no espaço; segundo, o padrão da chuva de sementes ao redor de cada planta-mãe, além da atividade de agentes dispersores; terceiro, o comportamento de forrageio de herbívoros comedores de sementes e de plântulas; e quarto, a distribuição espacial de locais adequados à germinação.

De acordo com Antonini e Nunes Freitas (2004), o padrão agregado de distribuição espacial é característico de espécies vegetais dispersas por animais. A canela-sassafrás apresenta dispersão por animais, denominada zoocoria, sendo que os grupos de animais citados como responsáveis por esse processo são principalmente aves, macacos e roedores (CARVALHO, 2005). A canela-sassafrás apresenta sementes recalcitrantes em relação ao armazenamento e dormência dupla, tegumentar e de embrião (CARVALHO, 2005). Nesse caso, aves e macacos são potenciais dispersores primários, já que as sementes são retiradas diretamente da planta-mãe e levadas a outros locais (MELLO, 2002). Provavelmente, os roedores encarregam-se da dispersão secundária da canela-sassafrás, realizando o transporte de sementes que estão sobre o solo.

Os animais dispersores têm grande influência sobre os padrões espaciais de sementes e, consequentemente, de plântulas (CRAWLEY, 1986). As aves, por exemplo, têm um comportamento alimentar ligado ao uso de poleiros naturais. Os roedores, ao invés de consumi-las no mesmo local, levam para abrigos de alimentação. Devido a esses comportamentos, as sementes contidas nos frutos são, em sua maioria, descartadas ou defecadas em tais locais, gerando um padrão de agregação secundária nas plântulas (MELLO, 2002). O grau de influência da dispersão das sementes depende também da maneira como estão distribuídas as plantas-mãe (CRAWLEY, 1986).

A presença de clareiras é um fator que favorece a agregação dos indivíduos jovens, devido ao aumento da intensidade de luz (CAPRETZ, 2004).

Cetnarski Filho (2003) analisou o grau de dispersão da regeneração de $O$. odorifera a partir do Índice de Morisita (Id), encontrando o padrão de dispersão agregado de conformidade com os resultados encontrados por Sidoruk Vidal (2000) em uma Floresta Estacional Semidecidual localizada no município de Cássia, MG. Essa característica parece ser comum ao gênero Ocotea, pois Caldoto et al. (2003) classificaram Ocotea puberula Nees (canela-guaicá) como sendo de dispersão agregada em todas as fases de sua vida.

O modelo de Janzen-Connell, segundo Crawley (1986), propõe sobrevivência máxima a certa distância da planta-mãe, e o contramodelo de Hubbell sugere que a mortalidade denso-dependente é compensada pela chuva de sementes perto da planta-mãe. Ambos os modelos sugerem agregação espacial de plântulas em diferentes graus.

\section{CONCLUSÕES}

- A distribuição de frequência dos diâmetros e alturas em classes caracterizou curvas na forma de Jinvertido, com um alto valor nas menores classes. Esses resultados sugerem que $O$. odorifera tem boa capacidade de se regenerar naturalmente e apresenta potencialidades para o manejo e conservação, o que refuta a hipótese da ela estar ameaçada de extinção por causas naturais. Há que se adotar, sim, medidas para o correto manejo sustentável da espécie.

- A espécie apresentou em geral um padrão espacial agregado para as classes de regeneração em todas as escalas analisadas, indicando um padrão característico da espécie nas fases iniciais. Os principais processos subjacentes foram associados à ocorrência de manchas de hábitat favorável, ocasionado pela heterogeneidade ambiental, além de outras hipóteses relacionadas à dispersão zoocórica por espécies com hábitos alimentares ligados a abrigos ou poleiros naturais.

- Tornam-se necessárias mais pesquisas para testar as hipóteses existentes acerca dos processos geradores, responsáveis pelos padrões observados na regeneração de $O$. odorifera. 
- O comportamento de mudança do padrão espacial agregado para padrão aleatório com o aumento do tamanho dos indivíduos sugere que a mortalidade e o recrutamento estão sendo determinados principalmente por fatores bióticos, como a competição intraespecífica e a mortalidade dependente de densidade.

- Devido às características estruturais apresentadas pela população de $O$. odorifera, a FLONA de Irati assume grande importância na conservação da espécie, pois foi encontrada uma alta densidade de indivíduos estabelecidos e um abundante banco de plântulas e indivíduos juvenis.

\section{AGRADECIMENTOS}

Ao Programa de Pós-Graduação em Ciências Florestais e ao Departamento de Engenharia Florestal da UNICENTRO, pela oportunidade e apoio para tornar este estudo possível. Ao Instituto Chico Mendes de Conservação da Biodiversidade - ICMBio, pela autorização concedida ( $\mathrm{n}^{\circ}$ 25871-1) para as atividades na FLONA de Irati. Ao professor Dr. Afonso Figueiredo Filho e demais colaboradores do Laboratório de Manejo Florestal, por disponibilizar os dados primários utilizados neste estudo. A todos que auxiliaram na coleta de dados em campo. À Capes, pela bolsa de mestrado.

\section{REFERÊNCIAS}

ANTONINI, R. D.; NUNES-FREITAS, A. F. Estrutura populacional e distribuição espacial de Miconia prasina DC. (Melastomataceae) em duas áreas de Floresta Atlântica na Ilha Grande, RJ, Sudeste do Brasil. Acta Bot. Bras. 18: p. 671 - 676. 2004.

AZAMBUJA, W. Óleos essenciais: O início de sua história no Brasil. Disponível em: <http://oleosessenciais.org/tag/safrol/>. Acesso em: 21/12/2011.

BADDELEY, A.; TURNER, R. Spatstat: an R package for analyzing spatial point patterns. Journal of Statistical Software, v. 12 (6), p. 1 - 42. 2005. Disponível em: <http://www.jstatsoft.org>. Acesso em: 16/05/2012.

BAILEY, T. C.; GATRELL, A. C. Interactive Spatial Data Analysis. New York, NY: John Wiley and Sons, $1995.413 \mathrm{p}$.

BARREIRO, E. J.; FRAGA, C. A. M. A utilização do safrol, principal componente químico do óleo de sassafrás, na síntese de substâncias bioativas na cascata do ácido araquidônico: anti-inflamatórios, analgésicos e antitrombóticos. Química Nova, v. 22, n. 5, p. 744 - 759, 1999.

BATISTA, J. L. F.; MAGUIRE, D. A. Modelling the spatial structure of tropical forests. Forest Ecology and Manegement, v. 110, p. 293 - 314, 1998.

BEGON, M.; TOWNSEND, C. R.; HARPER, J. L. Ecology: from individuals to ecosystems. 4. ed. Oxford: Blackwell, 2006. $738 \mathrm{p}$.

CALDOTO, L. S.; VERA. N.; DONAGH, P. M. Estructura poblacional de Ocotea puberula em um bosque secundário y primário de la selva mixta misionera. Ciência Florestal, Santa Maria, v. 13, n. 1, p. $25-32,2003$.

CAPRETZ, R. L. Análise dos padrões espaciais de árvores em quatro formações florestais do estado de São Paulo, através de análises de segunda ordem, como a função $\boldsymbol{K}$ de Ripley. 79 f. Dissertação (Mestrado em Ecologia de Agroecossistemas) - Escola Superior de Agricultura "Luiz de Queiroz", Piracicaba, 2004.

CARVALHO, P. E. R. Canela-sassafrás. Circular técnica, 110. Colombo: EMBRAPA Florestas, 2005. 12 p.

Espécies florestais brasileiras: recomendações silviculturais, potencialidades e uso da madeira. Colombo-PR: EMBRAPA-CNPF/SPI. 1994. 640 p.

Espécies florestais brasileiras. Brasília: Embrapa - Informação Tecnológica; Colombo-PR: Embrapa Florestas, 2003, v. 2, 1036 p. 
CETNARSKI FILHO, R. Regeneração natural de Ocotea Odorifera (Vell.) Rohwer (canela-sassafrás) em uma Floresta Ombrófila Mista no estado do Paraná. 79 f. Dissertação (Mestrado em Engenharia Florestal) - Universidade Federal do Paraná, Curitiba, PR, 2003.

CONDIT, R.; ASHTON, P.; BAKER, P.; BUNYAVEJCHEWIN, S.; GUNATILLEKE, S.; GUNATILLEKE, N.; HUBBELL, S. P.; FOSTER, R. B.; ITOH, A.; LAFRANKIE, J. V.; LEE, H. S.; LOSOS, E.; MANOKARAN, N.; SUKUMAR, R.; YAMAKURA, T. Spatial patterns in the distribution of tropical tree species. Science, v. 288, p. 1414 - 1418, 2000.

CRAWLEY, M. J. Plant Ecology. Oxford: Blackwell Scientific Publications, 1986. 496 p.

CRUZ, O. A. M.; RÍOS, R. V. Evaluación ecológica y silvicultural de ecosistemas boscosos. Ibagué, Colombia: Universidad del Tolima, 2003. 235 p.

DRUCK, S.; CARVALHO, M. S.; CÂMARA, G.; MONTEIRO, A. M. V. Análise espacial de dados geográficos. Brasília: EMBRAPA, 2004. 209 p.

DUBOIS, J. Los tipos de inventarios empleados en el manejo de los bosques tropicales, por sistemas naturales y seminaturales. Medellín, Col. Universidad Nacional de Colombia. 1980. 38 p.

EMBRAPA. Centro Nacional de Pesquisa de Solos (Rio de Janeiro, RJ). Sistema brasileiro de classificação de solos. Brasília: Embrapa Produção de Informação; Rio de Janeiro: Embrapa Solos, 1999. 412 p.

FIGUEIREIDO FILHO, A.; DIAS, A. N.; STEPKA, T. F.; SAWCZUK, A. R. Crescimento, mortalidade, ingresso e distribuição diamétrica em Floresta Ombrófila Mista. Floresta, v. 40, n. 4, p. 763 - 776, 2010.

GOODCHILD, M. F.; HAINING, R. P. GIS and spatial data analysis: covering perspectives. Papers in Regional Science, v. 83, p. 363 - 385, 2004.

HORVITZ, C. C.; LE CORFF, J. 1993. Spatial scale and dispersion pattern of ant and bird-dispersed herbs in two tropical lowland rain forests. Vegetatio, 107: $351-362$.

INOUE, M. T.; PUTTON, V. Macropropagação de 12 espécies florestais da Floresta Ombrófila Mista. Floresta, v. 37, n. 1, p. 55 - 61, 2007.

INOUE, M. T.; SCHRAIER, P. T.; DINIZ, M. R. Espécies florestais da Floresta Ombrófila Mista ameaçadas de extinção - canela-sassafrás (Ocotea odorifera (Vellozo) Rohwer). Revista da ALACS, v. 1, n. 1, p. 96 - 106, 2004.

INOUE, M. T.; RODERJAN, C. V.; KUNIYOSHI, Y. S. Projeto Madeira do Paraná. Curitiba: FUPEFUFPR, 1984. $260 \mathrm{p}$.

KEIL, S. S.; REISSMANN, C. B.; PELLICO NETTO, S. Crescimento e nutrição de Ocotea odorifera (canela-sassafrás) submetida a fertilização e a omissão de nutrientes. Pesquisa Florestal Brasileira, Colombo, PR, n. 58, p. 17 - 27, 2009.

LAW, R.; ILlian, J.; BURSLEM, D. F. R. P.; GRATZER, G.; GUNATILleKe, C. V. S.; GUNATILLEKE, I. Ecological information from spatial patterns of plants: insights from point process theory. Journal of Ecology, v. 97, n. 4, p. 616 - 628, 2009.

LEHN, C. R.; SALIS, S. M.; MATTOS, P. P.; DAMASCENO JÚNIOR, G. A. Estrutura e distribuição espacial de Trichilia elegans A. Juss. (Meliaceae) em uma floresta semidecídua no Pantanal da Nhecolândia, Mato Grosso do Sul, Brasil. Rev. Biol. Neotrop., v. 5(2), p. 1 - 9, 2008.

MATTEUCCI, W. G.; COLMA, A. Metodología para el estúdio de la vegetación. Washington: OEA, 1982. $168 \mathrm{p}$.

MAZZA, C. A. S. Caracterização ambiental da paisagem da Microrregião Colonial de Irati e zoneamento ambiental da Floresta Nacional de Irati, PR. 147 f. Tese (Doutorado em Ciências. Área Concentração em Ecologia e Recursos Naturais) - Universidade Federal de São Carlos, São Carlos, 2006. 
MELLO, M. A. R. de. Distribuição espacial de plantas em florestas Neotropicais. 15 f. Monografia (Disciplina: Ecologia de Populações de Plantas - NT238) - Universidade Estadual de Campinas, Campinas, 2002.

OLTRAMARI, A. C. Engenharia do metabolismo de células de canela-sassafrás (Ocotea odorifera (Vell.) Rohwer): análise da produção de biomassa celular e da síntese de safrol. 122 f. Dissertação (Mestrado) - Universidade Federal de Santa Catarina, Florianópolis, 2002.

PÉLLICO NETO, S.; FABROWSKI, F. J.; WEBER, S. H. Análise da estrutura diamétrica do sassafrás (Ocotea odorifera (Vell.) Rohwer) em fragmentos florestais no município de Fazenda Rio Grande, Paraná. Ambiência, Guarapuava, v. 3, n. 2, p. 167 - 181, 2007.

QUINET, A.; BAITELLO, J. B.; MORAES, P. L. R. de. Lauraceae in Lista de Espécies da Flora do Brasil. Jardim Botânico do Rio de Janeiro. Disponível em: <http://floradobrasil.jbrj.gov.br/2011/ FB008440>. Acesso em: 14/02/2012.

R Development Core Team (2012). R: A language and environment for statistical computing. R Foundation for Statistical Computing, Vienna, Austria. ISBN 3-900051-07-0. Disponível em: $<$ http://www.R-project.org/>.

RAMOS, P. H. X.; DALMASO, C. A.; NASCIMENTO, A. R. T.; MACEDO, A. F. Diversidade e estrutura diamétrica de um remanescente de Floresta Ombrófila Mista Aluvial no município de Lages, SC. In: SIMPÓSIO LATINO AMERICANO SOBRE MANEJO FLORESTAL, 4., 2008, Santa Maria. Anais... Santa Maria, 2008. p. 184.

REITZ, R.; KLEIN, R. M.; REIS, A. Projeto Madeira do Rio Grande do Sul. Sellowia. Itajaí, n. 34 35, p. 411 - 416, dez. 1983.

RIPLEY, B. D. Modeling spatial patterns (with discussion). Journal of the Royal Statistical Society, series B, v. 39, n. 2, p.172 - 212, 1977.

Spatial Statistics. New York: John Wiley and Sons, 1981. 252 p.

Statistical inference for spatial processes. Nova York: Cambridge University Press. 1988. 148 p.

RIZZINI, C. T. Árvores e madeiras úteis do Brasil: manual de dendrologia brasileira. São Paulo: Editora Edgard Blucher, 1981. 269 p.

SALOMÃO, A. N.; BRANDÃO, J. E. M. de S.; SILVA, J. A. da. Distribuição geográfica de seis espécies florestais como subsídio para a escolha de áreas futuras de conservação "in situ". Revista do Instituto Florestal, São Paulo, v. 4, pt. 4, p. 1199 - 1205, 1992.

SIDORUK VIDAL, M. A. Análise biométrica da regeneração natural de algumas espécies em uma Floresta Estacional Semidecidual localizada no Município de Cássia, MG. 104 f. Dissertação (Mestrado em Engenharia Florestal) - Universidade Federal do Paraná, Curitiba, 2000.

SILVA, K. E. da; MARTINS, S. V.; SANTOS, N. T.; RIBEIRO, C. A. A. S. Padrões espaciais de espécies arbóreas tropicais. In: MARTINS, S. V. (Ed.). Ecologia de florestas tropicais do Brasil. Viçosa: UFV, 2009. p. 216 - 244.

STERNER, R. W.; RIBIC, C. A.; SCHATZ, G. E. Testing for life historical changes in spatial patterns of four tropical tree species. Journal of Ecology, v.74, p. 621 - 633, 1986.

VATTIMO, I de. Contribuição ao conhecimento da distribuição geográfica das Lauraceae III. Rodriguésia, Rio de Janeiro, v. 31, n. 48, p. 7 - 57, 1979.

VELOSO, H. P.; RANGEL FILHO, A. L. R.; LIMA, J. C. A. Classificação da vegetação brasileira, adaptada a um sistema universal. Rio de Janeiro: Instituto Brasileiro de Geografia e Estatística, 1991. 124 p. 